\title{
Kirschen gegen Gichtattacken
}

Schmerzhafte Gichtattacken ließen sich in einer US-Studie mit 633 Gichtpatienten deutlich reduzieren, wenn die Patienten vorbeugend Kirschen aßen. So sank das Risiko für eine Attacke um 35\%, wenn die Teilnehmer an den zwei vorangegangenen Tagen Kirschen gegessen hatten. Mit zunehmender Menge ließ sich die Wirkung der roten Früchte steigern, optimal schienen täglich ca. 10-12 Kirschen.

Den Früchten werden antientzündliche und antioxidative Eigenschaften zugeschrieben, die auf ihrem hohen Gehalt an dem Pflanzenfarbstoff Anthocyan beruhen sollen. Sie sollen außerdem die Produktion von Harnsäure hemmen, bislang war jedoch nichts davon belegt.

Die Autoren der Studie wollen die Kirschtherapie nun in präventive Strategien einbinden. Weil die Therapie mit harnsäuresenkenden Medikamenten auf Patienten mit häufigen Gichtattacken, Gichttophi oder fortgeschrittener Krankheit beschränkt ist, würde die Kirschtherapie eine gute Alternative für Patienten in leichteren Stadien darstellen, so die Autoren.

\section{Hier steht eine Anzeige.}

\author{
Springer
}

\section{Vitamin D schützt Atemwege nicht}

Eine monatliche Vitamin-D-Supplementation von $100.000 \mathrm{IU}$ verringert Häufigkeit und Schwere von Infekten der oberen Atemwege nicht. Zu diesem Ergebnis kommen neuseeländische Wissenschaftler, die in ihrer doppelblinden, placebokontrollierten Studie mit 322 Teilnehmern sowohl jahreszeitliche Komponenten als auch den Serum-Basiswert für 25-Hydroxyvitamin D berücksichtigt haben. Damit bestätigt die Studie die Daten zweier früherer Studien. Übrigens bestehe die Möglichkeit, dass andere Bevölkerungsgruppen profitieren, etwa bei Vitamin-D-Mangel mit 25-OHD-Werten unter $10 \mathrm{ng} / \mathrm{ml}$. Auch eine andere Dosierungsweise könne möglicherweise zu abweichenden Resultaten führen. Diese Aspekte müssten in künftigen Studien untersucht werden. 